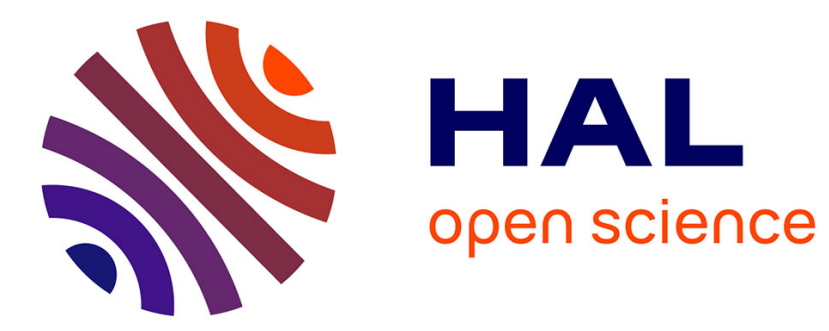

\title{
Dual Hyperquaternion Poincaré Groups
}

Patrick R. Girard, Patrick Clarysse, Romaric Pujol, Robert Goutte, Philippe

Delachartre

\section{To cite this version:}

Patrick R. Girard, Patrick Clarysse, Romaric Pujol, Robert Goutte, Philippe Delachartre. Dual Hyperquaternion Poincaré Groups. Advances in Applied Clifford Algebras, 2021, 31 (2), 10.1007/s00006021-01120-z . hal-03139597

\section{HAL Id: hal-03139597 \\ https://hal.science/hal-03139597}

Submitted on 28 Nov 2021

HAL is a multi-disciplinary open access archive for the deposit and dissemination of scientific research documents, whether they are published or not. The documents may come from teaching and research institutions in France or abroad, or from public or private research centers.
L'archive ouverte pluridisciplinaire HAL, est destinée au dépôt et à la diffusion de documents scientifiques de niveau recherche, publiés ou non, émanant des établissements d'enseignement et de recherche français ou étrangers, des laboratoires publics ou privés. 


\section{Advances in Applied Clifford Algebras \\ Dual Hyperquaternion Poincaré Groups \\ --Manuscript Draft--}

Manuscript Number:

Full Title:

Article Type:

Keywords:

Corresponding Author:
AACA-D-20-00060R2

Dual Hyperquaternion Poincaré Groups

Original Research

Quaternions; Hyperquaternions; Dual hyperquaternions; Poincaré groups; Canonical decomposition

Patrick Ralph Girard, Ph.D.

Universite de Lyon

FRANCE

\section{Corresponding Author Secondary}

Information:

Corresponding Author's Institution:

Universite de Lyon

Corresponding Author's Secondary Institution:

\section{First Author:}

First Author Secondary Information:

Order of Authors:

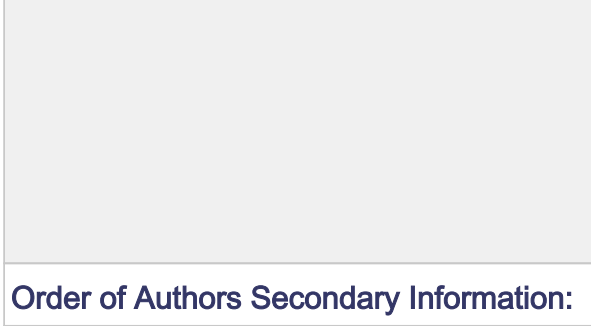

Funding Information:

\section{Abstract:}

Response to Reviewers:
Patrick Ralph Girard, Ph.D.

Patrick Ralph Girard, Ph.D.

Patrick Clarysse

Romaric Pujol

Robert Goutte

Philippe Delachartre

A new representation of the Poincaré groups in $\mathrm{n}$ dimensions via dual hyperquaternions is developed, hyperquaternions being defined as a tensor product of quaternion algebras (or a subalgebra thereof). This formalism yields a uniquely defined product and simple expressions of the Poincaré generators, with immediate physical meaning, revealing the algebraic structure independently of matrices or operators. An extended multivector calculus is introduced (allowing an eventual sign change of the metric or of the exterior product). The Poincaré groups are formulated as a dual extension of hyperquaternion pseudo-orthogonal groups. The canonical decomposition and the invariants are discussed. As concrete example, the 4D Poincare group is examined together with a numerical application. Finally, the hyperquaternion representation is compared to the quantum mechanical one. Potential applications include in particular, moving reference frames and computer graphics.

All reviewer comments have been taken into account. 


\title{
Dual Hyperquaternion Poincaré Groups
}

\author{
Patrick R. Girard, Patrick Clarysse, Romaric Pujol, Robert \\ Goutte and Philippe Delachartre
}

\begin{abstract}
A new representation of the Poincaré groups in $n$ dimensions via dual hyperquaternions is developed, hyperquaternions being defined as a tensor product of quaternion algebras (or a subalgebra thereof). This formalism yields a uniquely defined product and simple expressions of the Poincaré generators, with immediate physical meaning, revealing the algebraic structure independently of matrices or operators. An extended multivector calculus is introduced (allowing a possible sign change of the metric or of the exterior product). The Poincaré groups are formulated as a dual extension of hyperquaternion pseudo-orthogonal groups. The canonical decomposition and the invariants are discussed. As concrete example, the $4 D$ Poincaré group is examined together with a numerical application. Finally, the hyperquaternion representation is compared to the quantum mechanical one. Potential applications include in particular, moving reference frames and computer graphics.
\end{abstract}

Mathematics Subject Classification (2010). Primary 15A66; Secondary 11E88,15A67.

Keywords. Quaternions, Hyperquaternions, Dual hyperquaternions, Poincaré groups, Canonical decomposition.

\section{Introduction}

The $4 D$-Poincaré group is the group of linear transformations leaving invariant the Lorentz metric $d s^{2}=c^{2} d t^{2}-d x^{2}-d y^{2}-d z^{2}$ (with the signature $+---)$ and is constituted of rotations and space-time translations. This group is of great relevance in physics in particular, general relativity, relativistic quantum mechanics and particle physics, free particles being characterized by invariants of that group [1]. An important subgroup of the Poincaré group is the group of euclidean motions including rotations and translations in $3 D$ euclidean space. Two major methods for physical applications have been developed in that case, the homogeneous matrix transform and dual quaternions $[18,28]$. Generalizing the $4 D$-Poincaré group to the 
$n D$ case (with an arbitrary signature), we shall call them Poincaré groups. Various representations of the Poincaré groups have been proposed either in specific dimensions or signatures and are often expressed in terms of matrices $[4,5,7,8,16,19,21,24-27]$. Yet, matrices are neither the only nor probably the best way to represent rotation groups. An alternative is to use Clifford algebras in particular hyperquaternions defined as a tensor product of quaternion algebras (or a subalgebra thereof). Recently, we have applied hyperquaternions to the unitary, unitary symplectic and pseudo-orthogonal groups in $n$ dimensions $[9,10]$. Here, we propose to develop a dual hyperquaternion representation of Poincaré groups. The method yields simple expressions of the Poincaré generators and an efficient computation (algebraic or numerical), distinct from the matrix one. Furthermore, it relates the Poincaré groups to a new algebraic setting thereby opening new perspectives of unification. After a short introduction specifying the basic concepts and notation, an extended multivector calculus is presented (allowing a possible sign change of the metric or of the exterior product). Then we discuss the $n D$ Poincaré group, its algebra, a canonical decomposition into simple planes and the invariants. As concrete example, we study the $4 D$ Poincaré group, provide a numerical example and compare the hyperquaternion representation to the quantum mechanical one. Potential applications include in particular, moving reference frames and computer graphics.

\section{Background: Quaternions and Hyperquaternions}

We briefly introduce quaternions and hyperquaternions to specify the notations and basic concepts $[9,10,12]$. The quaternion algebra $\mathbb{H}$ is constituted by quaternions

$$
a=a_{1}+a_{2} i+a_{3} j+a_{4} k \quad\left(a_{i} \in \mathbb{R}\right)
$$

where $i, j, k$ satisfy the fundamental relations

$$
i^{2}=j^{2}=k^{2}=i j k=-1 \text {. }
$$

The quaternion conjugate is $a_{c}=a_{1}-a_{2} i-a_{3} j-a_{4} k$ with

$$
a a_{c}=a_{1}^{2}+a_{2}^{2}+a_{3}^{2}+a_{4}^{2}, \quad(a b)_{c}=b_{c} a_{c} .
$$

A hyperquaternion is a tensor product of quaternion algebras (or a subalgebra thereof). Thus, $\mathbb{H} \otimes \mathbb{H}$ is the tensor product of two quaternion algebras. Calling $(i, j, k)$ and $(I, J, K)$ two commuting quaternionic systems, one writes

$$
(i, j, k) \otimes 1=(i, j, k), \quad 1 \otimes(i, j, k)=(I, J, K)
$$

which uniquely specifies the tensor product. To define $\mathbb{H} \otimes \mathbb{H} \otimes \mathbb{H}$, one introduces a third quaternionic system $(l, m, n)$ commuting with the previous ones. Similarly, one obtains $\mathbb{H} \otimes \mathbb{H} \otimes \cdots \otimes \mathbb{H}$ (and the subalgebras $\mathbb{H} \otimes \mathbb{C}, \mathbb{H} \otimes \mathbb{H} \otimes \mathbb{C}$, etc.). Due to the isomorphism $\mathbb{H} \otimes \mathbb{H} \simeq m(4, \mathbb{R})$ where $m(4, \mathbb{R})$ stands for real square matrices of degree 4 , hyperquaternions yield all square real, complex and quaternionic matrices. A hyperconjugation $\mathbb{H}_{c} \otimes \mathbb{H}_{c} \otimes \cdots \otimes \mathbb{H}_{c}$ entails the transposition, adjunction and the transposition quaternion conjugate [10]. 
Whereas Hamilton viewed quaternions as a $3 D$ (if not $4 D$ ) system, Clifford, adopting Grassmann's ideas, considered quaternions as having only two generators $\left(e_{1}=i, e_{2}=j, e_{1} e_{2}=k, e_{1}^{2}=e_{2}^{2}=-1\right)$ suitable for a $2 D$ plane physics. He furthermore was the first to introduce tensor products of quaternion algebras, the concept of tensor product ("compounds of algebras") having been introduced a few years earlier. In his fundamental paper, Clifford proved that tensor products of quaternions constitute Clifford algebras [3]. A proof and an explicit expression of the generators is given in [10]. Lipschitz, shortly after and independently of Clifford, gave a simple expression of the $n$-dimensional euclidean rotation groups and thereby rediscovered the (even) Clifford algebra (constituted by products of an even number of generators) [20]. Moore, was to call Lipschitz's algebras hyperquaternions and developed a canonical decomposition (into simple orthogonal planes) thereof $[22,23]$. An extension of Moore's method to pseudo-euclidean rotations has recently been presented by the authors [9]. An advantage of the hyperquaternion formalism over the matrix one, is to yield physically meaningful parameters and straightforward computations. Moreover, besides rotations, hyperquaternions yield all unitary and unitary symplectic groups [10]. Mathematically, hyperquaternions (defined as tensor products of quaternion algebras) are Clifford algebras $C_{n}(p, q)$ having $n=p+q$ generators $e_{i}$ such that $e_{i} e_{j}+e_{j} e_{i}=0(i \neq j), e_{i}^{2}=+1$ ( $p$ generators $)$ and $e_{i}^{2}=-1$ ( $q$ generators $)$ where the generators are given in a compact hyperquaternionic form (for example $e_{1}=i K l$, etc.). Products of distinct generators yield multivectors in $V_{k},(0 \leq k \leq n)$ such as vectors $e_{i}\left(V_{1}\right)$, bivectors $e_{i} e_{j}(i<j)\left(V_{2}\right)$, trivectors $e_{i} e_{j} e_{k}(i<j<k)\left(V_{3}\right)$ etc.. $C_{n}^{+}(p, q)$ is the (even) subalgebra constituted by products of an even number of $e_{i}, C_{n}^{-}(p, q)$ is the rest of the algebra. The conjugate $A_{c}$ of a general element $A$ is obtained by replacing the $e_{i}$ by their opposite $-e_{i}$ and reversing the order of the elements

$$
\left(A_{c}\right)_{c}=A, \quad(A B)_{c}=\left(B_{c}\right)\left(A_{c}\right) .
$$

The commutator of two hyperquaternions is $[A, B]=\frac{1}{2}(A B-B A)$ and the dual of $A$ is $A^{*}=i_{d} A$ where $i_{d}=e_{1} \wedge e_{2} \wedge \cdots \wedge e_{n}$. The operations between the multivectors constitute the multivector calculus which we shall now examine.

\section{Extended Multivector Calculus}

As compared to the standard multivector calculus [2], we present here an extended multivector calculus allowing a possible sign change of the metric or the exterior product [10-13].

The interior and exterior products of two vectors $a\left(=\sum_{1}^{n} a_{i} e_{i}\right), b$ can be defined via the identity

$$
a b \equiv \frac{a b+b a}{2}+\frac{a b-b a}{2}=\lambda a \cdot b+\mu a \wedge b
$$


where $\lambda, \mu$ are two constant coefficients equal to \pm 1 (making possible a sign change of the metric or of the exterior product) with

$$
a . b=\lambda^{-1} \frac{a b+b a}{2}, \quad a \wedge b=\mu^{-1} \frac{a b-b a}{2} .
$$

Next, we consider products between a vector and a multivector. Given a multivector $A_{p}=a_{1} \wedge a_{2} \wedge \cdots \wedge a_{p}(2 \leq p<n)$ where $a_{p}$ are vectors, we define the interior product $[2]$

$$
a \cdot A_{p}=\sum_{k=1}^{p}(-1)^{k+1}\left(a . a_{k}\right) a_{1} \wedge \cdots \wedge a_{k-1} \wedge a_{k+1} \wedge \cdots \wedge a_{p} .
$$

The particular multivectors $a \wedge A_{2}, a \wedge A_{3}$ are defined via the relations

$$
a A_{2}=\frac{\lambda}{\mu}\left(a \cdot A_{2}\right)+a \wedge A_{2}, \quad a A_{3}=\lambda\left(a . A_{3}\right)+\mu a \wedge A_{3} .
$$

Generalized to a multivector $A_{p}(2 \leq p<n)$, the above relations become

$$
\begin{aligned}
& a A_{p}=\frac{\lambda}{\mu^{p-1}}\left(a \cdot A_{p}\right)+\mu^{p} a \wedge A_{p}, \\
& A_{p} a=\frac{\lambda}{\mu^{p-1}}\left(A_{p} \cdot a\right)+\mu^{p} A_{p} \wedge a .
\end{aligned}
$$

Postulating, a priori

$$
A_{p} \cdot a \equiv(-1)^{p-1} a \cdot A_{p}, \quad A_{p} \wedge a \equiv(-1)^{p} a \wedge A_{p},
$$

one derives from Eq. (3.6) after multiplication by $(-1)^{p}$ :

$$
(-1)^{p} A_{p} a=\frac{-\lambda}{\mu^{p-1}}\left(a \cdot A_{p}\right)+\mu^{p} a \wedge A_{p} .
$$

Combining Eqs. (3.5)-(3.8), the general formulas yield

$$
\begin{aligned}
2 a \cdot A_{p} & =\mu^{p-1} \lambda^{-1}\left[a A_{p}-(-1)^{p} A_{p} a\right], \\
2 a \wedge A_{p} & =\mu^{-p}\left[a A_{p}+(-1)^{p} A_{p} a\right]
\end{aligned}
$$

(giving the standard formulas for $\lambda=\mu=1$ ).

Interior and exterior products between multivectors are defined by

$$
\begin{array}{r}
A_{p} \wedge B_{q}=a_{1} \wedge\left(a_{2} \wedge \cdots \wedge a_{p} \wedge B_{q}\right), \\
A_{p} . B_{q}=\left(a_{1} \wedge \cdots \wedge a_{p-1}\right) \cdot\left(a_{p} \cdot B_{q}\right) \quad(p \leq q)
\end{array}
$$

with $A_{p} \cdot B_{q}=(-1)^{p(q+1)} B_{q} \cdot A_{p}[2]$. In particular, for bivectors $B_{i}$ one has

$$
B_{1} B_{2}=B_{1} \cdot B_{2}+B_{1} \wedge B_{2}+\left[B_{1}, B_{2}\right]
$$

yielding respectively a scalar, a tetravector and a bivector. These relations constitute the basic computational rules of the hyperquaternion algebras which we shall now apply to the Poincaré groups. 


\section{Poincaré Groups in $n$ Dimensions}

In this section, we develop a hyperquaternion representation of the Poincaré group in $n$ dimensions. To this effect, we embed the $n D$ space in an affine $(n+1) D$ space and express the Poincaré group as rotations and reflections in the affine space. We begin with the algebraic formalism followed by the canonical decomposition and the invariants.

\subsection{Algebraic Formalism}

Consider a hyperquaternion algebra $C_{n+1}\left(p^{\prime}, q^{\prime}\right)$ having $n+1\left(=p^{\prime}+q^{\prime}\right)$ generators (squaring to \pm 1 ) $e_{1}, e_{2}, \ldots, e_{n}, e_{n+1}$ and let $X$ be an element of an affine space

$$
X=e_{n+1}+\varepsilon x
$$

where $x$ belongs to the vector space $V_{1}$ with $x=\sum_{i=1}^{n} e_{i} x_{i}\left(x_{i} \in \mathbb{R}\right)$ and $\varepsilon$ commutes with all generators $\left(\varepsilon^{2}=0\right)$. The hyperquaternion algebra $C_{n}(p, q)$ associated with $V_{1}(n=p+q)$ has the metric (with $\lambda=\mu=1$ )

$$
d s^{2}=d x . d x=d x^{2}=\left(d x_{1}^{2}+\cdots+d x_{p}^{2}\right)-\left(d x_{p+1}^{2}+\cdots+d x_{p+q}^{2}\right) .
$$

A vector $x$ is timelike if $x . x>0$, spacelike if $x . x<0$ and isotropic if $x . x=0$. The Poincaré group is constituted by the isometries of this metric, i.e., the pseudo-orthogonal group $O(p, q)$ and translations which we shall consider successively.

The pseudo-orthogonal group $O(p, q)$ is generated by at most $n$ orthogonal symmetries. An orthogonal symmetry with respect to a plane (going through the origin) and perpendicular to a unit vector $u\left(u^{2}= \pm 1\right)$ is expressed by the formula (see Appendix A)

$$
x^{\prime}=\frac{u x u}{u u_{c}}
$$

with $x^{\prime 2}=x^{2}, u u_{c}=-u^{2}$. Hence, time and space like symmetries correspond respectively to

$$
x^{\prime}=-u x u \quad\left(u^{2}=1\right), \quad x^{\prime}=u x u \quad\left(u^{2}=-1\right) .
$$

Combining $r$ time and $s$ space symmetries one obtains the four types of pseudo-orthogonal transformations $A$ of $O(p, q)$ as indicated in Table 1. Sub-

TABLE 1. Hyperquaternion group $O(p, q)$ with $r$ time and $s$ space symmetries ( $e$ : even, o: odd)

\begin{tabular}{l|llll} 
component & $L_{+}^{\uparrow}$ & $L_{+}^{\downarrow}$ & $L_{-}^{\uparrow}$ & $L_{-}^{\downarrow}$ \\
\hline$(r, s)$ & $(e, e)$ & $(o, o)$ & $(e, o)$ & $(o, e)$ \\
$\operatorname{det} A$ & 1 & 1 & -1 & -1 \\
$x^{\prime}=$ & $a x a_{c}$ & $-a x a_{c}$ & $-a x a_{c}$ & $a x a_{c}$ \\
$a a_{c}=$ & 1 & -1 & 1 & -1 \\
$a \in$ & $C_{n}^{+}(p, q)$ & $C_{n}^{+}(p, q)$ & $C_{n}^{-}(p, q)$ & $C_{n}^{-}(p, q)$
\end{tabular}


groups of $O(p, q)$ are [27]

$$
\begin{gathered}
O(p, q)=L_{+}^{\uparrow} \cup L_{+}^{\downarrow} \cup L_{-}^{\uparrow} \cup L_{-}^{\downarrow}, \\
S O^{+}(p, q)=L_{+}^{\uparrow}, \quad S O(p, q)=L_{+}^{\uparrow} \cup L_{+}^{\downarrow} .
\end{gathered}
$$

Furthermore,

$$
L_{-}^{\downarrow}=e_{1} L_{+}^{\uparrow}, \quad L_{-}^{\uparrow}=e_{p+1} L_{+}^{\uparrow}, \quad L_{+}^{\downarrow}=e_{1} e_{p+1} L_{+}^{\uparrow}
$$

where $e_{1}, e_{p+1}$ can be replaced by other unit vectors of the same type. Thus, one has

$$
a=e_{1} a^{\prime}\left(a a_{c}=-1, a^{\prime} a_{c}^{\prime}=1, a \in C_{n}^{-}(p, q), a^{\prime} \in C_{n}^{+}(p, q)\right) \text { etc. }
$$

Embedding $C_{n}(p, q)$ in the algebra $C_{n+1}\left(p^{\prime}, q^{\prime}\right)$, the $O(p, q)$ group leaves the axis $e_{n+1}$ unchanged and can be expressed as

$$
X^{\prime}=a X a_{c}=e_{n+1}+\varepsilon x^{\prime}
$$

with $x^{\prime}= \pm a x a_{c}\left(a a_{c}= \pm 1, a \in C_{n+1}^{+}\left(p^{\prime}, q^{\prime}\right)\right.$ or $\left.C_{n+1}^{-}\left(p^{\prime}, q^{\prime}\right)\right)$.

A translation $T$ (in $V_{n}$ ) is given by

$$
X^{\prime}=b X b_{c}=e_{n+1}+\varepsilon(x+t)
$$

with

$$
b=e^{\varepsilon e_{n+1} \frac{t}{2}}=1+\varepsilon e_{n+1} \frac{t}{2}\left(b b_{c}=1, b \in C_{n+1}^{+}\left(p^{\prime}, q^{\prime}\right), e_{n+1}^{2}=-1\right)
$$

and $t=\sum_{i=1}^{n} e_{i} t_{i}, t_{i} \in \mathbb{R}$ (if $e_{n+1}^{2}=1$, one takes $b=e^{\varepsilon \frac{t}{2} e_{n+1}}$ ). Since $b b_{c}=1$ and $b \in C_{n+1}^{+}\left(p^{\prime}, q^{\prime}\right)$, a translation corresponds to a rotation of $S O_{n+1}^{+}$.

Combining the pseudo-orthogonal group $O(p, q)$ and the translations $T$, one obtains the full Poincaré group of Table 2 with the relations

TABLE 2. Hyperquaternion Poincaré group

\begin{tabular}{l|llll} 
component & $P_{+}^{\uparrow}$ & $P_{+}^{\downarrow}$ & $P_{-}^{\uparrow}$ & $P_{-}^{\downarrow}$ \\
\hline $\operatorname{det} A$ & 1 & 1 & -1 & -1 \\
$X^{\prime}=$ & $f X f_{c}$ & $-f X f_{c}$ & $-f X f_{c}$ & $f X f_{c}$ \\
$f f_{c}=$ & 1 & -1 & 1 & -1 \\
$f \in$ & $C_{n+1}^{+}\left(p^{\prime}, q^{\prime}\right)$ & $C_{n+1}^{+}\left(p^{\prime}, q^{\prime}\right)$ & $C_{n+1}^{-}\left(p^{\prime}, q^{\prime}\right)$ & $C_{n+1}^{-}\left(p^{\prime}, q^{\prime}\right)$ \\
$\qquad=P_{+}^{\uparrow} \cup P_{+}^{\downarrow} \cup P_{-}^{\uparrow} \cup P_{-}^{\downarrow}$, \\
$P_{-}^{\uparrow}=e_{p+1} P_{+}^{\uparrow}$, & $P_{-}^{\downarrow}=e_{1} P_{+}^{\uparrow}, \quad P_{+}^{\downarrow}=e_{1} e_{p+1} P_{+}^{\uparrow}$
\end{tabular}

where $P_{+}^{\uparrow}$ is the restricted Poincaré group; for its Lie algebra, see Appendix B. Our next step will be the canonical decomposition of the restricted Poincaré group. 


\subsection{Canonical Decomposition of the Restricted Group}

An element of the restricted Poincaré group $P_{+}^{\uparrow}$ being a rotation of $S O_{n+1}^{+}$, one can apply the canonical decomposition of pseudo-orthogonal rotations presented in [9]. To this effect, consider the algebra $C_{n+1}\left(p^{\prime}, q^{\prime}\right)$ having $n+1$ generators with $n=2 k$ (even) or $2 k+1$ (odd) and the Poincaré transform

$$
X^{\prime}=f X f_{c} \quad\left(f f_{c}=1, f \in C_{n+1}^{+}\left(p^{\prime}, q^{\prime}\right)\right) .
$$

The even (dual) hyperquaternion $f$ is of the type

$$
f=S+P+\frac{P \wedge P}{2 ! S}+\cdots+\frac{P \wedge P \wedge \cdots(m \text { factors })}{m ! S^{m-1}}
$$

where $S$ is the scalar part of $f$ and $P$ is a (dual) bivector. From $f$ one computes

$$
B=\frac{P}{S}=M+\varepsilon N
$$

with

$$
N=e_{n+1} \sum_{i=1}^{n} e_{i} \alpha_{i} \quad\left(\alpha_{i} \in \mathbb{R}\right),
$$

where $N$ is a simple plane $(N \wedge N=0)$ since all terms contain the vector $e_{n+1}$. The canonical decomposition $B=\sum_{i=1}^{m} b_{i} B_{i}$ yields at most $m=k+1$ orthogonal simple (dual) planes $B_{i}$

$$
B_{i}=M_{i}+\varepsilon N_{i}, \quad B_{i}^{2} \in\{ \pm 1,0\} .
$$

From $B_{i} \wedge B_{i}=0$, one obtains

$$
\left(M_{i}+\varepsilon N_{i}\right) \wedge\left(M_{i}+\varepsilon N_{i}\right)=M_{i} \wedge M_{i}+2 \varepsilon N_{i} \wedge M_{i}=0
$$

where we have used the commutativity of the exterior product of two bivectors; hence,

$$
M_{i} \wedge M_{i}=0, \quad N_{i} \wedge M_{i}=0
$$

which entails that $M_{i}$ is a simple plane and that $N_{i}$ belongs to the same plane and anticommutes with it $\left(N_{i} M_{i}=-M_{i} N_{i}\right)$. For $M_{i} \neq 0$, one has

$$
B_{i}^{2}=\left(M_{i}+\varepsilon N_{i}\right)\left(M_{i}+\varepsilon N_{i}\right)=M_{i}^{2}= \pm 1
$$

which for $B_{i}^{2}=-1\left(b_{i}=\tan \frac{\Phi_{i}}{2}\right)$ yields

$$
e^{\frac{\Phi_{i}}{2} B_{i}}=\cos \frac{\Phi_{i}}{2}+\left(M_{i}+\varepsilon N_{i}\right) \sin \frac{\Phi_{i}}{2}
$$

and for $B_{i}^{2}=1\left(b_{i}=\tanh \frac{\Phi_{i}}{2}\right)$

$$
e^{\frac{\Phi_{i}}{2} B_{i}}=\cosh \frac{\Phi_{i}}{2}+\left(M_{i}+\varepsilon N_{i}\right) \sinh \frac{\Phi_{i}}{2} .
$$

For $M_{i}=0$, one has a pure translation $e^{\varepsilon N_{i}}=1+\varepsilon N_{i}$. Finally, one obtains the algebraically compact decomposition

$$
f=e^{\frac{\Phi_{1}}{2} B_{1}} e^{\frac{\Phi_{2}}{2} B_{2}} \cdots e^{\frac{\Phi_{m}}{2} B_{m}} .
$$


For each component $f_{i}=e^{\frac{\Phi_{i}}{2} B_{i}}$, the rotation $R_{i}=e^{\frac{\Phi_{i}}{2} M_{i}}$ is known. Writing

$$
f_{i}=R_{i} T_{i} \quad\left(\text { or } T_{i} R_{i}\right)
$$

the translation $T_{i}$ is obtained as $T_{i}=R_{i}^{-1} f_{i}$, (or $f_{i} R_{i}^{-1}$ ). For the entire $f$, one has $f=f_{1} f_{2} \cdots f_{m}$ where the $f_{i}$ commute, hence,

$$
\begin{aligned}
f & =\left(R_{1} T_{1}\right)\left(R_{2} T_{2}\right) \cdots\left(R_{m} T_{m}\right) \\
& =\left(R_{1} R_{2} \cdots R_{m}\right)\left(T_{1} T_{2} \cdots T_{m}\right)=R T
\end{aligned}
$$

yielding the translation $T=R^{-1} f$. In the same way, one obtains

$$
\begin{aligned}
f & =\left(T_{1} R_{1}\right)\left(T_{2} R_{2}\right) \cdots\left(T_{m} R_{m}\right) \\
& =\left(T_{1} T_{2} \cdots T_{m}\right)\left(R_{1} R_{2} \cdots R_{m}\right)=T R
\end{aligned}
$$

and finally $T=f R^{-1}$.

\subsection{Invariants of the Restricted Group}

The Poincaré invariants of the restricted group $P_{+}^{\uparrow}$ are obtained as follows. The intersection of the simple plane $N$ with the space $V_{n}=\operatorname{span}\left(e_{1} \cdots e_{n}\right)$ is a vector $P$, parallel to $N$ giving the invariant $P^{2}$. Next, we consider the multivectors

$$
\begin{aligned}
W_{1} & =P \wedge(M+\varepsilon N)=P \wedge M, \\
W_{2} & =P \wedge b_{1}\left(M_{1}+\varepsilon N_{1}\right) \wedge b_{2}\left(M_{2}+\varepsilon N_{2}\right) \\
& =\left(b_{1} b_{2}\right) P \wedge M_{1} \wedge M_{2}, \\
W_{k-1} & =\left(b_{1} b_{2} \cdots b_{k-1}\right) P \wedge M_{1} \wedge M_{2} \wedge \cdots \wedge M_{k-1}
\end{aligned}
$$

yielding the invariant inner products

$$
\left(W_{1} \cdot W_{1}\right), \quad\left(W_{2} \cdot W_{2}\right), \quad \ldots, \quad\left(W_{k-1} \cdot W_{k-1}\right) .
$$

If the dimension of the space is even $(n=2 m)$, one thus obtains $k-1$ invariants and with $P^{2}$, a total of $k$ invariants. If the dimension of the space is odd $(n=2 k+1)$, one has the $k$ invariants above plus the pseudo-scalar

$$
W_{m}=\left(b_{1} b_{2} \cdots b_{k}\right) P \wedge M_{1} \wedge M_{2} \wedge \cdots \wedge M_{k}
$$

which is an invariant by itself. As concrete example, we shall now examine the $4 D$ Poincaré group.

\section{Example: 4D Poincaré Group}

The $4 D$-Poincaré group is of central importance in physics, in particular in relativistic quantum mechanics and general relativity $[1,14,15]$. We shall first present the algebraic formulation, then a numerical application with a canonical decomposition and the invariants. Finally, we shall compare the hyperquaternion representation with the quantum mechanical and octonionic operator representations. 


\subsection{Algebraic Formulation}

Consider the hyperquaternion algebra $\mathbb{H} \otimes \mathbb{H} \otimes \mathbb{C}\left(\simeq C_{5}(2,3)\right)$ having five generators (see Appendix C)

$$
e_{0}=i J, \quad e_{1}=i K l, \quad e_{2}=i K m, \quad e_{3}=i K n, \quad e_{4}=i I .
$$

The metric of the algebra $C_{4}(1,3)\left(e_{4}=0\right)$ is

$$
d s^{2}=d x . d x=d x^{2}=d x_{0}^{2}-d x_{1}^{2}-d x_{2}^{2}-d x_{3}^{2}
$$

$\left(x=\sum_{i=0}^{3} e_{i} x_{i}, x_{i} \in \mathbb{R}\right)$. The restricted Poincaré group $P_{+}^{\uparrow}$ is composed of spatial rotations, hyperbolic rotations (boosts) and space-time translations which are respectively given by a total of ten generators (each of the two first equations below yield three generators and the third one four)

$$
\begin{aligned}
e^{\frac{\theta}{2} B} & =\cos \frac{\theta}{2}+\sin \frac{\theta}{2} B \quad\left[B^{2}=-1, B \in(l, m, n)\right], \\
e^{\frac{\Phi}{2} B} & =\cosh \frac{\Phi}{2}+\sinh \frac{\Phi}{2} B \quad\left[B^{2}=1, B \in(I l, I m, I n)\right], \\
e^{\varepsilon \frac{\lambda}{2} B} & =1+\varepsilon \frac{\lambda}{2} B \quad\left[B^{2}=( \pm 1,0), B \in(K, J l, J m, J n), \lambda \in \mathbb{R}\right] .
\end{aligned}
$$

The combination of these transformations generates the element $f$ :

$$
X^{\prime}=f X f_{c} \quad\left(f f_{c}=1, f \in C_{5}^{+}(2,3)\right)
$$

$\left(X=i I+\varepsilon x, X^{\prime}=i I+\varepsilon x^{\prime}\right)$. The canonical decomposition of $f$ leads to at most two simple orthogonal planes $B_{i}$ :

$$
\begin{aligned}
& B=b_{1} B_{1}+b_{2} B_{2}=M+\varepsilon N, \\
& f=e^{\frac{\Phi_{1}}{2} B_{1}} e^{\frac{\Phi_{2}}{2} B_{2}} \quad\left(B_{i}^{2}= \pm 1,0\right) .
\end{aligned}
$$

The projection of the bivector $N$ on the space $V_{4}=\operatorname{span}\left(e_{0} e_{1} e_{2} e_{3}\right)$ gives a vector $P$ and the invariant $P^{2}$ which can be positive, negative or nil. The second invariant is $\left(W_{1} \cdot W_{1}\right)$ with $W_{1}=P \wedge M$. In the following, we shall provide a numerical example to illustrate the procedure.

\subsection{Numerical Example}

As numerical example of a canonical decomposition, we consider the product of a spatial rotation followed by a translation and a boost leading to the element $f$ of the $4 D$-Poincaré transform $X^{\prime}=f X f_{c}$ :

$$
\begin{aligned}
f & =e^{\frac{\Phi_{2}}{2} m I} e^{\varepsilon(-2 J l+K)} e^{\frac{\Phi_{1}}{2} m} \\
& =(2+\sqrt{3} m I)[1+\varepsilon(-2 J l+K)]\left(\frac{\sqrt{3}}{2}+\frac{m}{2}\right)
\end{aligned}
$$

$\left(\tan \frac{\Phi_{1}}{2}=\frac{1}{\sqrt{3}}=b_{1}, \tanh \frac{\Phi_{2}}{2}=\frac{\sqrt{3}}{2}=b_{2}\right)$. The canonical decomposition [9] leads to the expression

$$
f=e^{\frac{\Phi_{2}}{2} B_{2}} e^{\frac{\Phi_{1}}{2} B_{1}}
$$


where $\Phi_{1}, \Phi_{2}$ have the same values as above and $B_{1}, B_{2}$ are two simple orthogonal commuting (dual) planes $\left(B_{1}^{2}=-1, B_{2}^{2}=1\right)$

$$
B_{1}=m-2 \varepsilon J(\sqrt{3} l+n), \quad B_{2}=m I+\varepsilon\left(-J m+\frac{2}{\sqrt{3}} K\right) .
$$

The relativistic invariants are obtained as follows. From the relation $B=$ $M+\varepsilon N\left(=b_{1} B_{1}+b_{2} B_{2}\right)$, one finds

$$
M=m\left(\frac{1}{\sqrt{3}}+\frac{\sqrt{3}}{2} I\right), \quad N=-J\left(2 l+\frac{\sqrt{3}}{2} m+\frac{2}{\sqrt{3}} n\right)+K .
$$

The orthogonal projection of the plane $N$ on the 4 -space $V_{4}=\operatorname{span}\left(e_{0} e_{1} e_{2} e_{3}\right)$ $(=\operatorname{span}(I))$ gives a vector $P$ via the formula

$$
\begin{aligned}
P=N^{*} \cdot V_{4}=(i N) \cdot V_{4} & =\frac{i N I-I i N}{2}=-i I N \\
& =i K\left(2 l+\frac{\sqrt{3}}{2} m+\frac{2}{\sqrt{3}} m\right)+i J
\end{aligned}
$$

and the first invariant $P^{2}=-\frac{61}{12}$. To get the other one, one computes

$$
\begin{aligned}
W_{1}=P \wedge M & =\frac{P M+M P}{2} \\
& =i\left(-J l+\frac{J m}{\sqrt{3}}+\sqrt{3} i J n-\frac{K}{2}\right) \in V_{3},
\end{aligned}
$$

yielding the second invariant

$$
W_{1} \cdot W_{1}=-\frac{49}{12}=P^{2}\left(M_{\perp}\right)^{2}
$$

where $M_{\perp}$ is the component of $M$ perpendicular to $P[12]$

$$
\begin{aligned}
M_{\perp} & =P^{-1}(P \wedge M) \\
& =\frac{1}{61}(24 l-\sqrt{3} m-8 \sqrt{3} n-10 I l+32 \sqrt{3} m I-14 \sqrt{3} I n)
\end{aligned}
$$

and $M_{\perp}^{2}=\frac{49}{61}$. The Clifford dual of the three-vector $W_{1}$ is the vector $W$

$$
W=I W_{1}=i\left(-K l+\frac{K m}{\sqrt{3}}+\sqrt{3} i K n+\frac{J}{2}\right)
$$

giving the same invariant $W^{2}=W_{1}^{2}$ (with $I=e_{0} e_{1} e_{2} e_{3}$ ). The vector $W$ plays a similar role as the Pauli-Lubanski vector in quantum mechanics. This numerical example illustrates the fact that the dual hyperquaternion formulation completely reveals the abstract algebraic properties of the Poincaré group making it perhaps more accessible than other representations. 


\subsection{Other Representation}

Other representations of the $4 D$-Poincaré group exist. In quantum mechanics, the translations and rotations (spatial and hyperbolic) are represented respectively by the operators

$$
\widehat{P_{\mu}}=\frac{\partial}{\partial x^{\mu}}, \quad \widehat{M_{\mu \nu}}=x^{\mu} \frac{\partial}{\partial x^{\nu}}-x^{\nu} \frac{\partial}{\partial x^{\mu}}
$$

acting on a spin- 0 wave function with the mass as invariant. For a spin- $1 / 2$ Dirac wave function, the Poincaré generators are

$$
\widehat{P_{\mu}}=\frac{\partial}{\partial x^{\mu}}, \quad \widehat{M_{\mu \nu}}=x^{\mu} \frac{\partial}{\partial x^{\nu}}-x^{\nu} \frac{\partial}{\partial x^{\mu}}-\frac{1}{4}\left[\gamma_{\mu}, \gamma_{\nu}\right]
$$

where $\gamma_{\mu}$ are the Dirac matrices with the anticommutator $\left\{\gamma_{\mu}, \gamma_{\nu}\right\}=-2 g_{\mu \nu}$ having as invariants the mass and the spin [1]. Both representations have the same Lie algebra as the hyperquaternion representation, the latter being however spin independent (see Appendix B). The hyperquaternion representation thus constitutes a new form of representation (with hyperquaternion generators) distinct from the quantum mechanical one, revealing the abstract algebraic structure of the Poincaré group. It is to be noticed that dual hyperquaternions lead for certain Poincaré groups to a unitary representations $(\mathbb{H} \otimes \mathbb{H} \otimes \mathbb{C} \simeq m(4, \mathbb{C}))$ and to unitary symplectic ones for others $(\mathbb{H} \otimes \mathbb{H} \otimes \mathbb{H} \simeq m(4, \mathbb{H}))$.

Another Poincaré representation, developed for the standard model, makes use of a tensor product of the four division algebras $\mathbb{R} \otimes \mathbb{C} \otimes \mathbb{H} \otimes \mathbb{O}$ where $(1)$ stands for the octonion algebra which is related to quaternions $[6,17]$. Though this algebra is neither a Clifford algebra nor associative, it shares with the hyperquaternionic approach the idea that physics might result from algebra and in particular from tensor products of algebras. Yet, since groups and group representations are associative, operators have to be constructed which seem to be isomorphic to the complex Clifford algebra $C_{6}(\mathbb{C})$ leading to the isomorphisms

$$
C_{6}(\mathbb{C}) \simeq m(8, \mathbb{C}) \simeq m(4, \mathbb{R}) \otimes m(2, \mathbb{C}) \simeq(\mathbb{H} \otimes \mathbb{H}) \otimes(\mathbb{H} \otimes \mathbb{C}) .
$$

Hence, in the end, it seems that the octonionic approach is compatible with the hyperquaternionic one.

Though Poincaré groups are very important, they do not constitute the largest covariant group of physics. Thus Maxwell's equations in vacuum are covariant with respect to the conformal group which contains the Poincaré group as subgroup. Is it possible to express the conformal groups as hyperquaternions? This will be the object of a next study.

\section{Conclusion}

The paper has given a new dual hyperquaternion representation of Poincaré groups in $n$ dimensions distinct from the matrix one. The formalism yields simple expressions of the Poincaré generators, with immediate physical meaning. After the introduction of an extended multivector calculus, the algebraic 
formalism of the Poincaré groups has been developed as well as the canonical decomposition and invariants. As example, the $4 D$-Poincaré group and a numerical example have been examined. Finally, the hyperquaternion representation has been compared to the quantum mechanical one. It is hoped that the dual hyperquaternion representation might deepen the abstract algebraic understanding of the Poincaré groups and provide a new compact, efficient computational tool. Potential applications include in particular, moving reference frames and computer graphics.

\section{Acknowledgment}

This work was supported by the LABEX PRIMES (ANR-11-LABX-0063) and was performed within the framework of the LABEX CELYA (ANR10-LABX-0060) of Université de Lyon, within the program "Investissements d'Avenir" (ANR-11-IDEX-0007) operated by the French National Research Agency (ANR).

The authors gratefully acknowledge the comments of the journal referees which have greatly improved the readability of the paper.

\section{Appendix A. Orthogonal Plane Symmetry}

For the convenience of the reader, we derive here the formula of Eq. (4.3) $[12,13]$. The orthogonal symmetric $x^{\prime}$ of a vector $x$ with respect to a plane orthogonal to a vector $u$ satisfies the equations

$$
x^{\prime}=x+k u, \quad u \cdot\left(\frac{x^{\prime}+x}{2}\right)=0(k \in \mathbb{R}) .
$$

Hence,

$$
\begin{gathered}
u \cdot\left(x+\frac{k u}{2}\right)=0 \Rightarrow k=\frac{-2 u \cdot x}{u \cdot u}, \\
x^{\prime}=x-\frac{2(u \cdot x)}{u \cdot u}=x-\frac{(u x+x u) u}{u u}=\frac{u x u}{u u_{c}} .
\end{gathered}
$$

\section{Appendix B. Lie Algebra of the $n D$-Poincaré Group}

We first give the Lie algebra of the restricted Poincaré group $P_{+}^{\uparrow}$ and then of the full group $P$.

\section{B.1. Restricted Group}

Consider an $n D$ space imbedded in an $n+1$ hyperquaternion algebra having the generators $e_{1}, e_{2}, \ldots, e_{n}, e_{n+1}$. The Lie generators of the rotations are

$$
M_{i j}=\frac{1}{2} e_{i} e_{j} \quad(1 \leq i, j \leq n, i<j) .
$$


The Lie commutator being defined as $[A, B]=A B-B A$, one obtains for $i \neq j=r \neq s$ and

$$
\begin{aligned}
{\left[M_{i j}, M_{r s}\right] } & =\frac{1}{4}\left(e_{i} e_{j} e_{r} e_{s}-e_{r} e_{s} e_{i} e_{j}\right) \\
& =\frac{1}{4}\left(e_{i} e_{j} e_{r} e_{s}+e_{i} e_{r} e_{j} e_{s}\right) \\
& =\frac{1}{2} \eta_{j r} e_{i} e_{s}=\eta_{j r} M_{i s}
\end{aligned}
$$

with $\eta_{j r}=\left(e_{j} e_{r}+e_{r} e_{j}\right) / 2$. Similarly, one has

$$
\begin{aligned}
& {\left[M_{i j}, M_{r s}\right]=\eta_{i s} M_{j r} \quad(j \neq i=s \neq r),} \\
& {\left[M_{i j}, M_{r s}\right]=-\eta_{j s} M_{i r} \quad(i \neq j=s \neq r),} \\
& {\left[M_{i j}, M_{r s}\right]=-\eta_{i r} M_{j s} \quad(j \neq i=r \neq s) ;}
\end{aligned}
$$

combining all possible cases for the rotations one gets

$$
\left[M_{i j}, M_{r s}\right]=\eta_{j r} M_{i s}+\eta_{i s} M_{j r}-\eta_{j s} M_{i r}-\eta_{i r} M_{j s} .
$$

For the $n D$-translations, the generators are

$$
M_{(n+1) i}=\frac{1}{2} \varepsilon e_{n+1} e_{i} \quad\left(1 \leq i \leq n, \varepsilon^{2}=0, e_{n+1}^{2}=-1\right)
$$

(for $e_{n+1}^{2}=1$, the one takes $\left.M_{i(n+1)}=-M_{(n+1) i}\right)$. One has the relations

$$
\left[M_{(n+1) i}, M_{(n+1) j}\right]=0 \quad(\forall i, j)
$$

and for $i \neq j=k$

$$
\begin{aligned}
{\left[M_{i j}, M_{(n+1) k}\right] } & =\frac{\varepsilon}{4}\left(e_{i} e_{j} e_{(n+1)} e_{k}-e_{(n+1)} e_{k} e_{i} e_{j}\right) \\
& =\frac{\varepsilon e_{n+1}}{4}\left(e_{i} e_{j} e_{k}+e_{k} e_{j} e_{i}\right) \\
& =\frac{\varepsilon e_{n+1}}{2} \eta_{j k} e_{i}=\eta_{j k} M_{(n+1) i}
\end{aligned}
$$

similarly, for $k=i \neq j$, one has

$$
\left[M_{i j}, M_{(n+1) k}\right]=-\eta_{i k} M_{(n+1) j} .
$$

Combining the two cases above, one obtains for the translations

$$
\left[M_{i j}, M_{(n+1) k}\right]=\eta_{j k} M_{(n+1) i}-\eta_{i k} M_{(n+1) j} .
$$

Projecting the plane $M_{(n+1) i}$ on the space $V_{n}$ one obtains, for $e_{n+1}^{2}=-1$, the vector

$$
P_{i}=e_{n+1} M_{(n+1) i}=\frac{\varepsilon}{2} e_{n+1} e_{n+1} e_{i}=-\frac{\varepsilon}{2} e_{i} .
$$

For $e_{n+1}^{2}=+1$, one has

$$
P_{i}=e_{n+1} M_{i(n+1)}=\frac{\varepsilon}{2} e_{n+1} e_{i} e_{n+1}=-\frac{\varepsilon}{2} e_{i} .
$$


The complete Lie algebra of the restricted Poincaré group can thus be expressed in the standard abstract form

$$
\begin{gathered}
{\left[M_{i j}, M_{r s}\right]=\eta_{j r} M_{i s}+\eta_{i s} M_{j r}-\eta_{j s} M_{i r}-\eta_{i r} M_{j s}} \\
{\left[P_{i}, P_{j}\right]=0} \\
{\left[M_{i j}, P_{k}\right]=\eta_{j k} P_{i}-\eta_{i k} P_{j} .}
\end{gathered}
$$

\section{B.2. Full Group}

The other components of the full Poincaré group being obtained from the restricted one through multiplication by a vector $e_{k}$, one has besides the above relations the following ones

$$
\begin{aligned}
{\left[M_{i j}, e_{k}\right] } & =\eta_{j k} e_{i}-\eta_{i k} e_{j}, \\
{\left[M_{(n+1) i}, e_{k}\right] } & =\eta_{i k}\left(\varepsilon e_{n+1}\right) .
\end{aligned}
$$

\section{Appendix C. Multivector Structure of $\mathbb{H} \otimes \mathbb{H} \otimes \mathbb{C}$}

The hyperquaternion algebra $\mathbb{H} \otimes \mathbb{H} \otimes \mathbb{C} \simeq C_{5}(2,3)$ can be viewed as an eightdimensional vector space over the (real) quaternion algebra with the basis $\{1, l, m, n\}$. Thus, any hyperquaternion $A$ can be written as a linear combination of eight linearly independent basis elements $(1, I, J, K, i, i I, i J, i K)$ with quaternionic coefficients $\left[q_{s}\right]=a_{s}+b_{s} l+c_{s} m+d_{s} n(1 \leq s \leq 8)$ as

$$
A=\left[q_{1}\right]+I\left[q_{2}\right]+J\left[q_{3}\right]+K\left[q_{4}\right]+i\left[q_{5}\right]+i I\left[q_{6}\right]+i J\left[q_{7}\right]+i K\left[q_{8}\right] .
$$

The multivector structure of $A$ can be derived from Eq. (5.1) as follows:

$I=e_{0123}, J=e_{1234}, K=e_{04}, l=e_{23}, m=-e_{13}, n=e_{12}, i=-e_{01234}$

where $e_{0123}=e_{0} e_{1} e_{2} e_{3}$, etc.. Then, the product of two elements $A, B$ can be implemented (algebraically or numerically) in Mathematica with its quaternion product, e.g., $\left[q_{i}\right] * *\left[q_{j}\right]$. For the convenience of the reader, the complete multivector structure is given in the table below.

$$
\left[\begin{array}{llll}
1 & l=e_{2} e_{3} & m=e_{3} e_{1} & n=e_{1} e_{2} \\
I=e_{0} e_{1} e_{2} e_{3} & I l=e_{1} e_{0} & I m=e_{2} e_{0} & I n=e_{3} e_{0} \\
J=e_{1} e_{4} e_{2} e_{3} & J l=e_{4} e_{1} & \mathrm{Jm}=e_{4} e_{2} & \mathrm{Jn}=e_{4} e_{3} \\
K=e_{0} e_{4} & K l=e_{0} e_{4} e_{2} e_{3} & K m=e_{4} e_{0} e_{1} e_{3} & K n=e_{0} e_{4} e_{1} e_{2} \\
i=e_{0} e_{4} e_{1} e_{2} e_{3} & i l=e_{4} e_{0} e_{1} & i m=e_{4} e_{0} e_{2} & i n=e_{4} e_{0} e_{3} \\
i I=e_{4} & i I l=e_{4} e_{2} e_{3} & i I m=e_{1} e_{4} e_{3} & i I n=e_{4} e_{1} e_{2} \\
i J=e_{0} & i J l=e_{0} e_{2} e_{3} & i J m=e_{1} e_{0} e_{3} & i J n=e_{0} e_{1} e_{2} \\
i K=e_{1} e_{2} e_{3} & i K l=e_{1} & i K m=e_{2} & i K n=e_{3}
\end{array}\right]
$$

\section{References}

[1] Adler, S.L.: Quaternionic Quantum Mechanics and Quantum Fields. Oxford University Press, New York (1995)

[2] Casanova, G.: L'algèbre vectorielle. PUF, Paris (1976) 
[3] Clifford, W.K.: Applications of Grassmann's extensive algebra. Amer. J. Math. 1, 266-276 (1878)

[4] Cnops, J.: An Introduction to Dirac Operators on Manifolds. Birkhäuser, Basel (2002)

[5] Costa, C., Tenser, M.R., Amorim, R.G.G., Fernandes, M.C.B., Santana, A.E., Vianna, J.D.M: Symplectic field theories: Scalar and spinor representations. Adv. Appl. Clifford Algebras 28, 27 (2018)

[6] Dray, T., Manogue, C.A.: The Geometry of Octonions. World Scientific (2015)

[7] Elstrodt, J., Grunewald, F., Mennicke, J.: Kloosterman sums for Clifford Algebras and a lower bound for the positive eigenvalues of the Laplacian for congruence subgroups acting on hyperbolic spaces. Inventiones mathematicae 101, 641-685 (1990)

[8] Elstrodt, J., Grunewald, F., Mennicke, J.: Vahlen's group of Clifford matrices and spin-groups. Mathematische Zeitschrift 196, 369-390 (1987)

[9] Girard, P.R., Clarysse, P., Pujol, R., Goutte, R., Delachartre, P.: Hyperquaternions: An efficient mathematical formalism for geometry. In: Nielsen F., Barbaresco F. (eds), Geometric Science of Information 2019. Lecture Notes in Computer Science 11712, 116-125, Springer, Cham (2019) https://doi.org/10.1007/978-3-030-26980-7-13

[10] Girard, P.R., Clarysse, P., Pujol, R., Goutte, R., Delachartre, P.: Hyperquaternions: A new tool for physics. Adv. Appl. Clifford Algebras 28, 68 (2018)

[11] Girard, P.R., Clarysse, P., Pujol, R., Wang, L., Delachartre, P.: Differential geometry revisited by biquaternion Clifford algebra. In J.-D. Boissonnat et al. (Eds.): Curves and Surfaces. Springer (2015)

[12] Girard, P.R.: Quaternions, Clifford Algebras and Relativistic Physics. Birkhäuser, Basel (2007)

[13] Girard, P.R.: Algèbre de Clifford et Physique relativiste. PPUR, Lausanne (2004)

[14] Girard, P.R.: Einstein's equations and Clifford algebra. Adv. Appl. Clifford Algebras 9, 2, 225-230 (1999)

[15] Girard, P.R.: Histoire de la Relativité Générale d'Einstein: Développement Conceptuel de la Théorie. Sciences de l'Homme et Société. University of Wisconsin-Madison USA (1981). https://hal.archives-ouvertes.fr/tel-02321688

[16] Goodman, R., Wallach, N.R.: Symmetry, Representations and Invariants. Springer, New York (2009)

[17] Gording, B., Schmidt-May, A.: The unified standard model. Adv. Appl. Clifford Algebras 30, 55 (2020)

[18] Hladik, J., Hladik, P-E.: Quaternions réels, duaux et complexes. Ellipses, Paris (2016)

[19] Karakus, S.O.: Screw theory in Lorentzian space. Adv. Appl. Clifford Algebras 29, 3 (2019)

[20] Lipschitz, R.: Principes d'un calcul algébrique qui contient comme espèces particulières le calcul des quantitiés imaginaires et des quaternions. C.R. Acad. Sci. Paris 91, 619-621, 660-664 (1880)

[21] Lounesto, P.: Clifford Algebras and Spinors. Cambridge University Press, Cambridge (2001) 
[22] Moore, C.L.E.: Hyperquaternions. J. Math. Phys. 1, 63-77 (1922)

[23] Moore, C.L.E.: Rotations in hyperspace. In: Proceedings of the American Academy of Arts and Sciences 53, 8, 651-694 (1918)

[24] Ohnuki, Y.: Unitary Representations of the Poincaré Group and Relativistic Wave Equations. World Scientific, Singapore (1988)

[25] Porteous, I.: Clifford Algebras and the Classical Groups. Cambridge University Press, Cambridge (1995)

[26] Saar, R., Groote, S.: Mass, zero nass and ... nophysics. Adv. Appl. Clifford Algebras 27, 2739-2768 (2017)

[27] Traubenberg, M.R.: Clifford algebras in physics. Adv. Appl. Clifford Algebras 19, 869-908 (2009)

[28] Wang, X., Zhu, H.: On the comparisons of unit dual quaternion and homogeneous transformation matrix. Adv. Appl. Clifford Algebras 24, 213-229 (2014)

Patrick R. Girard

Univ Lyon, INSA-LYON, Université Claude Bernard Lyon 1, UJM-Saint Etienne, CNRS, Inserm, CREATIS UMR 5220, U1206, F-69621 LYON, France

Bât Blaise Pascal

7 avenue Jean Capelle

F-69621 Villeurbanne, France

e-mail: patrick.girard@creatis.insa-lyon.fr

Patrick Clarysse

Univ Lyon, INSA-LYON, Université Claude Bernard Lyon 1, UJM-Saint Etienne, CNRS, Inserm, CREATIS UMR 5220, U1206, F-69621 LYON, France

Bât Blaise Pascal

7 avenue Jean Capelle

F-69621 Villeurbanne, France

Romaric Pujol

Pôle de Mathématiques, INSA-Lyon

Bât. Léonard de Vinci, 21 avenue Jean Capelle

F-69621 Villeurbanne, France

Robert Goutte

Univ Lyon, INSA-LYON, Université Claude Bernard Lyon 1, UJM-Saint Etienne, CNRS, Inserm, CREATIS UMR 5220, U1206, F-69621 LYON, France

Bât Blaise Pascal

7 avenue Jean Capelle

F-69621 Villeurbanne, France

Philippe Delachartre

Univ Lyon, INSA-LYON, Université Claude Bernard Lyon 1, UJM-Saint Etienne, CNRS, Inserm, CREATIS UMR 5220, U1206, F-69621 LYON, France

Bât Blaise Pascal

7 avenue Jean Capelle

F-69621 Villeurbanne, France 